\title{
Adaptive Generalized Spatial Modulation Algorithm Based on Joint Optimization
}

\author{
Fatang Chen, Fanchao Zha* and Hanyan Zhang \\ Chongqing Key Laboratory of Mobile Communications, Chongqing University of \\ Posts and Telecommunications, Chongqing 400065, China \\ zhafanchao123@sina.com
}

\begin{abstract}
An adaptive generalized spatial modulation (AGSM) algorithm based on joint optimization is proposed in this paper. First, the signal constellation and the spatial constellation are jointly designed to construct the generalized spatial modulation constellation. Then, the receiver selects the optimal constellation according to the channel state information, and feeds the optimal constellation information back to the sender. In the next transmission, the optimal generalized spatial modulation constellation is used for bit mapping. Furthermore, in order to reduce the computational complexity of AGSM algorithm, a local optimization algorithm is also proposed. Our simulation results show that the proposed algorithms can effectively improve the bit error ratio (BER) performance with low complexity and small feedback compared to the conventional GSM system.
\end{abstract}

Keywords: Adaptive spatial modulation; spatial constellation; channel state information; antenna combinations

\section{Introduction}

As a new multiple-input multiple-output (MIMO) technology, spatial modulation $(\mathrm{SM})$ has received extensive attention in the field of wireless communication [1-3]. In the conventional SM, only a single antenna is activated to transmit data, and thus the transmission rate is obviously lower than the spatial multiplexing technology in the traditional MIMO system.

In order to solve this defect of SM technology, the generalized spatial modulation (GSM) is proposed. As an extension of the SM technology, GSM can activate multiple antennas to transmit the modulation symbols in each time slot, and antenna combinations and modulation symbols together to transmit information. Compared to the SM technology, GSM systems can further improve the spectral efficiency. At present, the GSM technology mainly has two different implementation schemes: the single-stream transmission scheme, where all active antennas emit the same modulation symbol [4]; and the multiple-stream transmission scheme, in which different activate antennas transmit different symbols [5]. The former can be regarded as a diversity technology, which can be used to improve the system's bit error ratio (BER) performance. However, the latter is the combination of spatial multiplexing and SM technology, which can improve the spectral efficiency of SM system and will be the one considered in this work. Therefore, GSM can make a better compromise between the spectral efficiency and BER performance.

In the recent years, link adaptation (LA) technology has been gradually extended to the SM field. In [6-9], several antenna selection (TAS) technologies have been proposed based on various criteria, such as capacity optimized antenna selection (COAS) and Euclidean distance optimized antenna selection (EDAS). Since EDAS is better than COAS in improving the performance of BER system, the current researches tend to EDAS. To further exploit the spatial freedom of the MIMO channel, adaptive spatial 
modulation (ASM) technologies have been proposed in the literature of SM [10-13]. In ASM, the receiving end selects the optimal symbol modulation order for possible active antenna according to the channel state information (CSI) [10]. Theoretical analysis and simulation results show that it can effectively improve the BER performance of SM, but the algorithm is extremely complex. In order to reduce computational complexity of ASM, a candidate-reduction-based ASM (CR-ASM) is proposed in [11], which can effectively reduce the search space by removing the candidates with low probability. Though the closed-loop spatial modulation scheme can effectively utilize the channel state information (CSI) to improve the BER performance of system, the most of the adaptive technologies still are used for SM and few of them are involved in the GSM system.

Against of the background, this paper proposes an adaptive generalized spatial modulation algorithm (AGSM) to improve the BER performance of GSM system. In this proposed algorithm, spatial constellation and signal constellation firstly are combined to construct the generalized spatial modulation constellation. Then, receiver selects the optimal constellation according to the channel state information. At the end, the optimal constellation information is fed back to the sender and is used for bit mapping in the next transmission. Furthermore, considering the high computational complexity of the AGSM algorithm, a low complexity adaptive generalized spatial modulation algorithm (L-AGSM) is proposed. Simulation results shown the proposed algorithms can efficiently improve the BER performance of GSM.

\section{System Model}

Consider a GSM-MIMO system with $N_{T}$ antennas at the transmitter, and $N_{R}$ antennas at the receiver, the system block diagram is shown in Figure 1. Assumed that $N_{A}$ transmit antennas are activated to transmit modulation symbols in each time slot. Therefore, there are total $N=C_{N_{T}}^{N_{A}}$ antenna combinations. According to the bit mapping scheme of GSM, the information bits are first divided into two parts, the first $\log _{2} N_{C}$ bits are used to select antenna combinations. The second part is used for MPSK / MQAM modulation, which modulates $N_{A} \log _{2} M$ bits to form the symbol vector $\mathbf{s}=\left[s_{1}, s_{2}, \ldots, s_{N_{A}}\right]^{T}$, where $s_{i} \in \Omega, i=1,2, \ldots, N_{A}$, and $\Omega$ is the symbol modulation constellation. Then the modulate symbols will be transmitted on each activated antennas, and we can obtain the transmit signal vector $\mathbf{x}=\left[\ldots, 0, s_{1}, 0, \ldots, 0, s_{2}, 0, \ldots, 0, s_{N_{A}}, 0, \ldots\right]^{T}$, in which there are $N_{A}$ nonzero values and $N_{T}-N_{A}$ zero values. 


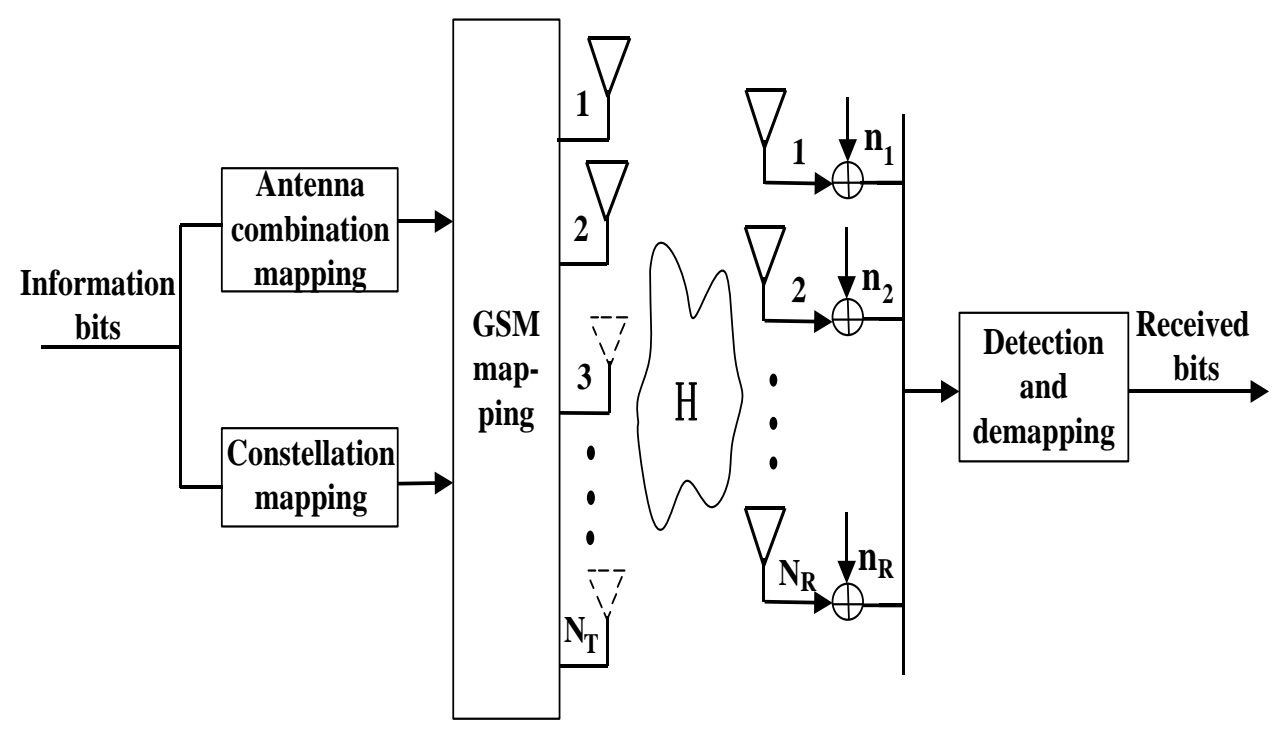

Figure 1. Block Diagram of the GSM-MIMO System

Assumed that the transmit signal vector $\mathbf{x}$ is transmitted through the $N_{R} \times N_{T}$ wireless MIMO channel $\mathbf{H}$. The $N_{R} \times 1$ received signal vector $\mathbf{y}$ can be expressed as

$$
\mathbf{y}=\mathbf{H x}+\mathbf{n}
$$

where $\mathbf{n}$ is $N_{R} \times 1$ additive white Gaussian noise vector. Each element of $\mathbf{H}$ and $\mathbf{n}$ follows a complex Gaussian distribution with zero mean and a variance of one. The expression of (1) can be further simplified as

$$
\mathbf{y}=\mathbf{H}_{k} \mathbf{s}+\mathbf{n}=\sum_{i=1}^{i=N_{A}} \mathbf{h}_{i} s_{i}+\mathbf{n}
$$

where $\mathbf{H}_{k}=\left[\mathbf{h}_{1}, \mathbf{h}_{2}, \ldots, \mathbf{h}_{N_{A}}\right]$ is the $N_{A} \times 1$ equivalent channel matrix, excluding the column of $\mathbf{H}$ which corresponding to non-active antennas. Therefore, the ML detection algorithm of GSM-MIMO system can be written as follows

$$
(\hat{k}, \hat{\mathbf{s}})=\arg \min _{k \in I, \mathbf{s} \in S}\left\|\mathbf{y}-\mathbf{H}_{k} \mathbf{s}\right\|_{F}^{2}
$$

where I represents the antenna combination index set, and $S$ represents the modulate symbol vector set.

\section{Adaptive Generalized Spatial Modulation Algorithm Based on Joint Optimization}

In [10], ASM scheme selects the optimum modulation order for each transmit antenna according to the channel state information (CSI) when a channel condition changes slowly. After that, the corresponding index is fed back to the sender. Finally, the sender uses the selected modulation order for the next transmission. Simulation results have shown that the adaptive mechanism can decrease the error possibility of bits. So in this paper, we also exploit the advantage of adaptive modulation to improve the BER performance of GSM. 


\subsection{Adaptive Generalized Spatial Modulation}

By the previous introduction of GSM, we know that the number of antenna combinations which will be used must be a power of two. So the set of the possible antenna combinations always contains redundant elements.

In this paper, a novel adaptive generalized spatial modulation method (AGSM) is proposed. In the proposed AGSM scheme, the antenna combinations and symbol modulation constellations are combined to form spatial modulation constellation point set. Then, spatial modulation constellations are constructed by selecting $N_{C}$ spatial modulation constellation points from the spatial modulation constellation point set. At the end, the receiver computes optimum spatial modulation constellation according to the CSI, and sends this information back to the transmitter.

For the purpose of description, the following two basic concepts are used in this article. The first basic concept is the spatial modulation constellation, which can be defined as

$$
\Delta_{q}=\left[q ; \Phi(q, 1), \Phi(q, 2), \ldots, \Phi\left(q, N_{C}\right)\right] \quad q=1,2, \ldots, Q
$$

where $\Phi(q, i)$ is the ith spatial modulation constellation point in the qth spatial modulation constellation, $i=1,2, \ldots, N_{C}$. The second basic concept is the spatial modulation constellation point, which can be expressed as

$$
\Phi(q, i)=\left[\Omega^{q}(i, 1), \Omega^{q}(i, 2), \ldots, \Omega^{q}\left(i, N_{T}\right)\right]
$$

where $\Omega^{q}(i, j)$ represents the symbol modulation scheme used at the $j$ th transmit antennas in the ith spatial modulation constellation point, $j=1,2, \ldots, N_{T} \cdot \Omega^{q}(i, j)=0$ denotes the corresponding transmit antenna is non-active, and $\Omega^{q}(i, j)=1$ denotes the corresponding transmit antenna is active but doesn't send modulation symbol.

In ASM, different active antennas may use different modulation orders to generate modulation symbol, so the number of information bits are transmitted may be also different in each time slot. When receiver wrongly detect the index of active antenna, it may lead to the number of received bits isn't same with the number of transmitted bits. In subsequent time slots, even the transmitted bits are detected correctly at the receiver, the received bit sequence is still different from the transmitted bit sequence. This phenomenon is called bit misalignment. To overcome this problem, the number of transmitted bits must be same in each time slot. So in the AGSM scheme, the point number of symbol modulation constellation in the ith and $\hat{i}$ th spatial modulation constellation point must meet

$$
\sum_{j=1}^{N_{T}} \log _{2} M^{q}(i, j)=\sum_{j=1}^{N_{T}} \log _{2} M^{q}(\hat{i}, j)=\eta-\log _{2} N_{C}
$$

where $M^{q}(i, j)$ represents the number of constellation points in the symbol modulation constellation which be used at the $j$ th transmit antenna in the ith spatial modulation constellation point. Compared with GSM system, the proposed AGSM scheme doesn't randomly select $2^{\left\lfloor\log _{2}\left(C_{N_{T}}^{N_{A}}\right\rfloor\right.}$ antenna combinations as antenna combinations set, but forms a spatial modulation constellation point set. The number of spatial modulation constellation points $N_{S}$ is determined according to spectral efficiency $\eta$ and antenna configuration. After the value of $N_{S}$ is determined, the number of alternative spatial modulation constellations $Q=C_{N_{S}}^{N_{C}}$ can be obtained. In particular, the information bits which be mapped by antenna combinations are used to select a spatial modulation constellation point in ASGM. Apparently, the number of candidate spatial modulation constellation is determined according to a given spectral efficiency and system antenna configuration. It should be noted that different active antennas can use different modulation schemes in the 
proposed AGSM. But in order to avoid bit the misalignment phenomenon which has described in the above, the available modulation schemes should guarantee the number of transmitted bits are same in each symbol interval.

As shown in [10], we instead use the nearest neighbor approximation for performance metric. Assume that the channel inputs are emitted with equal probability. The nearest neighbor approximation of the pairwise error probability (PEP) for a given channel $\mathbf{H}$ can be expressed as

$$
P\left(\mathbf{x}_{i} \rightarrow \mathbf{x}_{j} \mid \mathbf{H}\right)=\lambda \cdot Q\left(\sqrt{\frac{1}{2 N_{0}} d_{\min }^{2}(\mathbf{H})}\right)
$$

where $\lambda$ represents the average, umber of neighbor points, and $Q(\bullet)$ is complementary error function which can be denote as

$$
Q(x)=\frac{1}{\sqrt{2 \pi}} \int_{x}^{\infty} e^{-t^{2} / 2} d t
$$

Also, $N_{0}$ is the variance of noise, $d_{\min }(\mathbf{H})$ is the received minimum distance which is defined as

$$
d_{\text {min }}(\mathbf{H})=\min _{\substack{\mathbf{x}_{i} \neq \mathbf{x}_{j} \\ \mathbf{x}_{i}, \mathbf{x}_{j} \in \Psi}}\left\|\mathbf{H}\left(\mathbf{x}_{i}-\mathbf{x}_{j}\right)\right\|_{F}
$$

where $\Psi$ is transmitted signal vector set, $\mathbf{x}_{i}, \mathbf{x}_{j}$ respectively denotes two different signal vector. We can see from (7), PEP is a monotonically decreasing function of $d_{\min }(\mathbf{H})$. Hence, the system's BER performance may be improved by maximizing the received distance $d_{\min }(\mathbf{H})$.

Take the GSM system which has two active antennas as an example to elaborate the calculation process of $d_{\min }(\mathbf{H})$. For the qth spatial modulation constellation, the formula (9) can be expanded as

$$
\begin{aligned}
& d_{\min }^{q}(\mathbf{H})=\min _{\substack{a_{i j}^{k}, l_{i}^{k} \in \Phi(q, k) \\
b_{j 1}^{l}, b_{j 2}^{k} \in \Phi(q, l)}} \|\left(\begin{array}{c}
a_{i 1}^{k} h_{1, i 1}+a_{i 2}^{k} h_{1, i 2} \\
a_{11}^{k} h_{2, i 1}+a_{i 2}^{k} h_{2, i 2} \\
\vdots \\
a_{i 1}^{k} h_{N_{R}, i 1}+a_{i 2}^{k} h_{N_{R}, i 2}
\end{array}\right)-\left(\begin{array}{c}
b_{j 1}^{l} h_{1, j 1}+b_{j 2}^{l} h_{1, j 2} \\
b_{j 1}^{l} h_{2, j 1}+b_{j 2}^{l} h_{2, j 2} \\
\vdots \\
b_{j 1}^{l} h_{N_{R}, j 1}+b_{j 2}^{l} h_{N_{R}, j 2}
\end{array} \|_{F}\right.
\end{aligned}
$$

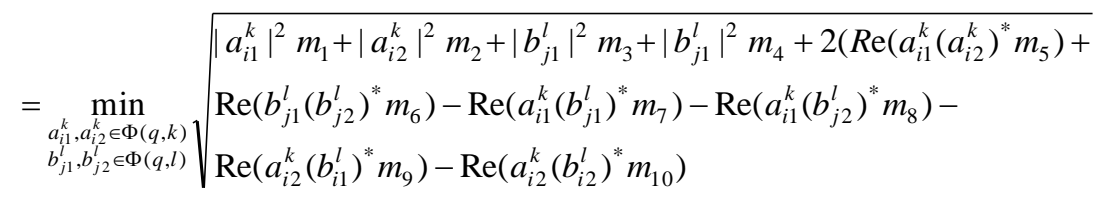

where $a_{i 1}^{k}, a_{i 2}^{k}$ denote modulation symbols transmitted at the 1 st and $2 n d$ active antenna in the $k t h$ spatial modulation constellation point, respectively. Likewise, $a_{i 1}^{k}, a_{i 2}^{k}$ denote modulation symbols transmitted at the $1 s t$ and $2 n d$ active antenna in the lth spatial modulation constellation point, respectively. $m_{1}, m_{2}, \ldots, m_{10}$ are the inner product of the columns of channel matrix, which are denoted as 


$$
\left\{\begin{array}{c}
m_{1}=\left|h_{1, i 1}\right|^{2}+\left|h_{2, i 1}\right|^{2}+\cdots+\left|h_{N_{R}, i 1}\right|^{2} \\
m_{2}=\left|h_{1, i 1}\right|^{2}+\left|h_{2, i 1}\right|^{2}+\cdots+\left|h_{N_{R}, i 1}\right|^{2} \\
m_{3}=\left|h_{1, j 1}\right|^{2}+\left|h_{2, j 1}\right|^{2}+\cdots+\left|h_{N_{R}, j 1}\right|^{2} \\
m_{4}=\left|h_{1, j 2}\right|^{2}+\left|h_{2, j 2}\right|^{2}+\cdots+\left|h_{N_{R}, j 2}\right|^{2} \\
m_{5}=h_{1, i 1} h_{1, i 2}^{*}+h_{2, i 1} h_{2, i 2}^{*}+\cdots+h_{N_{R}, i 1} h_{N_{R}, i 2}^{*} \\
m_{6}=h_{1, j 1} h_{1, j 2}^{*}+h_{2, j 1} h_{2, j 2}^{*}+\cdots+h_{N_{R}, j 1} h_{N_{R}, j 2}^{*} \\
m_{7}=h_{1, i 1} h_{1, j 1}^{*}+h_{2, i 1} h_{2, j 1}^{*}+\cdots+h_{N_{R}, i 1} h_{N_{R}, j 1}^{*} \\
m_{8}=h_{1, i 1} h_{1, j 2}^{*}+h_{2, i 1} h_{2, j 2}^{*}+\cdots+h_{N_{R}, i 1} h_{N_{R}, j 2}^{*} \\
m_{9}=h_{1, i 2} h_{1, j 1}^{*}+h_{2, i 2} h_{2, j 1}^{*}+\cdots+h_{N_{R}, i 2} h_{N_{R}, j 1}^{*} \\
m_{10}=h_{1, i 2} h_{1, j 2}^{*}+h_{2, i 2} h_{2, j 2}^{*}+\cdots+h_{N_{R}, i 2} h_{N_{R}, j 2}^{*}
\end{array}\right.
$$

Without loss of generality, the power of each modulation symbol is assumed to be normalized, namely, $\left|a_{i 1}^{k}\right|^{2}=\left|a_{i 2}^{k}\right|^{2}=\left|b_{j 1}^{l}\right|^{2}=\left|a_{j 2}^{l}\right|^{2}=1$. The $d_{\text {min }}$ (H) given by (10) can be simplified as

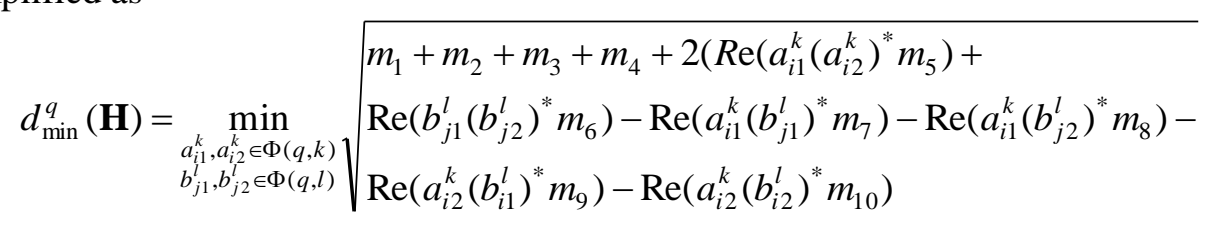

Assumed that $\Delta_{\text {opt }}$ denotes the optimal spatial modulation constellation. In AGSM, $\Delta_{\text {opt }}$ can be obtained by the following formula

$$
\begin{aligned}
\Delta_{\text {opt }} & =\underset{\Delta \in \Theta}{\arg \max } d_{\min }(\mathbf{H}) \\
& =\underset{\Delta \in \Theta}{\arg \max }\left(\min _{\substack{\mathbf{x}_{i}, \mathbf{x}_{j} \in \Psi_{\Delta} \\
\mathbf{x}_{i} \neq \mathbf{x}_{j}}}\left\|\mathbf{H}\left(\mathbf{x}_{i}-\mathbf{x}_{j}\right)\right\|_{F}\right)
\end{aligned}
$$

where $\Theta$ is the set of spatial modulation constellation. According the calculation way of (10), it need to first compute the minimum Euclidean distance of all spatial modulation constellation. Then, we choose the spatial modulation constellation which minimum Euclidean distance is maximum as the optimal spatial modulation constellation, Apparently, the calculation way has a high computational complexity.

For understanding the propose algorithm, it is necessary to take an example to explain the principle of AGSM. Assumed that a GSM system has four transmit antennas in which two transmit antennas are activated, and the spectral efficiency is $\eta=4 \mathrm{bit} / \mathrm{s} / \mathrm{Hz}$. Apparently, there are six antenna combinations. When the number of transmitted information bits are same, the modulation scheme for two active antennas has three types, and can be denote (BPSK,BPSK)、(QPSK,1)、(1,QPSK), respectively. So we can obtain $N_{S}=18$ spatial modulation constellation points and are shown in Table 1. 


\section{Table 1. The Set of Spatial Modulation Constellation Points}

\begin{tabular}{ccccc}
\hline $\begin{array}{c}\text { Modulation } \\
\text { constellation } \\
\text { point index }\end{array}$ & Antenna 1 & Antenna 2 & Antenna 3 & Antenna 4 \\
1 & BPSK & BPSK & 0 & 0 \\
2 & BPSK & 0 & BPSK & 0 \\
3 & BPSK & 0 & 0 & BPSK \\
4 & 0 & BPSK & BPSK & 0 \\
5 & 0 & BPSK & 0 & BPSK \\
6 & 0 & 0 & BPSK & BPSK \\
7 & QPSK & 1 & 0 & 0 \\
8 & QPSK & 0 & 1 & 0 \\
9 & QPSK & 0 & 0 & 1 \\
10 & 0 & QPSK & 0 & 1 \\
11 & 0 & QPSK & 1 & 0 \\
12 & 0 & 0 & QPSK & 1 \\
13 & 1 & QPSK & 0 & 0 \\
14 & 1 & 0 & QPSK & 1 \\
15 & 1 & 0 & 0 & QPSK \\
16 & 0 & 1 & QPSK & 0 \\
17 & 0 & 1 & 0 & QPSK \\
18 & 0 & 0 & 1 & QPSK \\
\hline
\end{tabular}

Figure 2 shows the principle block diagram of the AGSM system. Firstly, the system constructs $Q=C_{N_{S}}^{N_{C}}$ spatial modulation constellations and each contains $N_{C}$ spatial modulation constellation points which are selected from the set of spatial modulation constellation point. Then the optimal spatial modulation constellation is selected according to the channel state information at the receiving end, and is fed back to the sender. At the end, the sender uses the optimal spatial modulation constellation to map bits in the next transmission according to the feedback information. Simulation results show that AGSM can obtain better BER performance compared with GSM.

For the AGSM system which has been described in above, there are total $C_{18}^{4}=3060$ spatial modulation constellations. In order to obtain the minimum Euclidean distance $d_{\min }^{q}(\mathbf{H})$, we need to calculate four times Euclidean distance for the qth spatial modulation constellation. Thus, 12240 Euclidean distance value need be calculated for the AGSM algorithm. Obviously, the computational complexity of proposed algorithm is very high. 


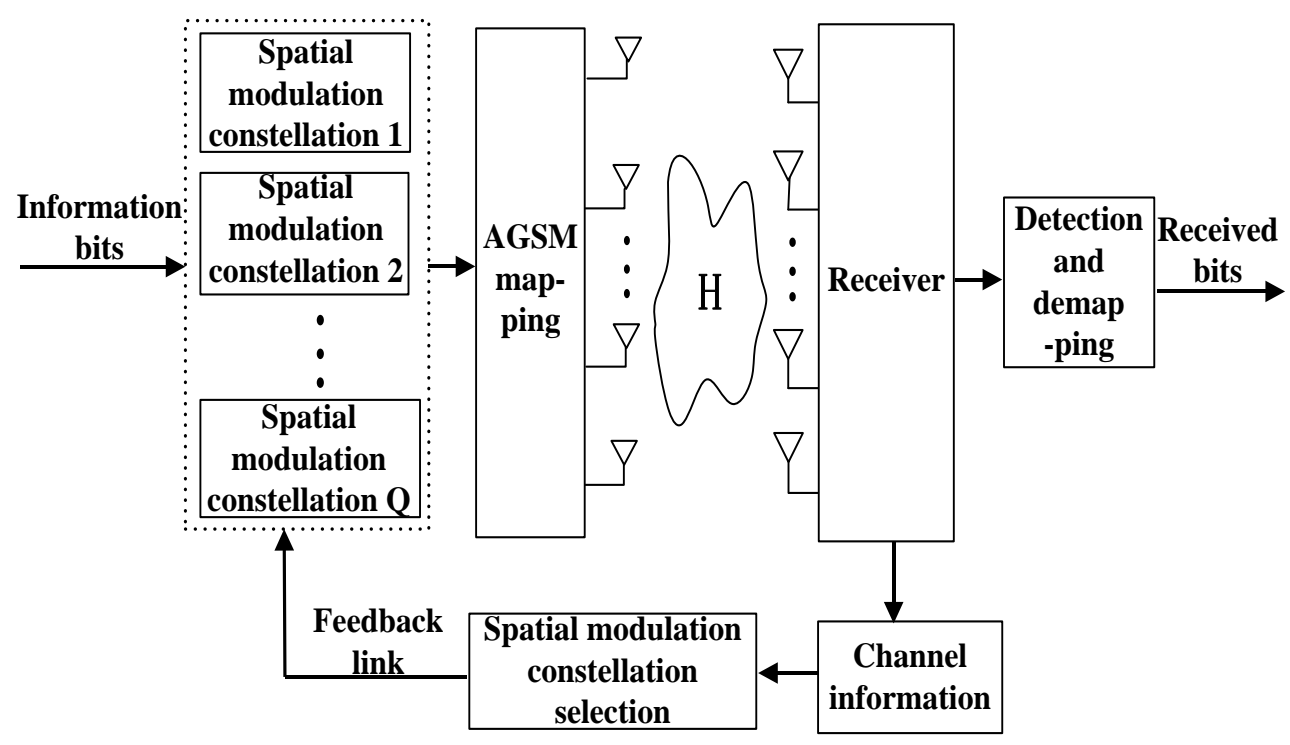

Figure 2. The Principle Block Diagram of AGSM

\subsection{Low Complexity AGSM Algorithm}

In order to reduce the calculation times of Euclidean distance in AGSM, a novel low complexity algorithm is proposed in this section. The proposed simplified algorithm divides the calculation process of optimal spatial modulation constellation into $N_{C}$ times, and obtain a local optimal spatial modulation constellation $\Delta_{i}^{\text {opt }}$ in each time, $i=1,2, \ldots, N_{S}$. Finally, we compare the minimum Euclidean distance of all local optimal spatial modulation constellations, and select the $\Delta_{i}^{o p t}$ which has maximum minimum Euclidean distance as the optimal spatial modulation constellation. Here, we use $\Gamma_{i}$ to denote the set which are composed of reminding spatial modulation constellation point, and initialize loop variable $i$ with 1 .Let $j$ be the number of spatial modulation constellation point in $\Delta_{i}^{o p t}$. The specific steps of the simplified algorithm are stated as follows

(1)Initialization

a. $j=1$

b. Initialize $\Delta_{i}^{\text {opt }}$ with the ith spatial modulation constellation point. Namely, there is only a spatial modulation constellation point in $\Delta_{i}^{o p t}$ at the beginning .

c. Initialize $\Gamma_{i}$ with reminding spatial modulation constellation points.

(2)The selection of $\Delta^{\text {opt }}$

a. Calculate the minimum Euclidean distance between the spatial modulation constellation points in $\Gamma_{i}$ and the spatial modulation constellation points in $\Delta_{i}^{o p t}$, which can be denoted as

$$
\Phi_{o p t}=\underset{\Phi \in \Gamma_{i}, \Phi \notin \Delta_{i}^{o p t}}{\arg } \max d_{\min }\left(\Phi, \Delta_{i}^{o p t}\right)
$$

where $\Phi_{o p t}$ represents the spatial modulation constellation point corresponding maximum minimum Euclidean distance, and belongs to $\Gamma_{i}$, not to $\Delta_{i}^{o p t}$.

b. Put the spatial modulation constellation point $\Phi_{o p t}$ into $\Delta_{i}^{o p t}$, and delete $\Phi_{o p t}$ from set $\Gamma_{i}, j=j+1$. If the number of spatial modulation constellation point $j<N_{C}$, then 
back to (2).Otherwise, record the spatial modulation constellation points in $\Delta_{i}^{o p t}$ and save the minimum Euclidean distance $d_{\min }^{i}(\mathbf{H})$.

c. Let $i=i+1$.If $i<=N_{S}$, then back to (1). Otherwise, go to (3).

(3)The selection of $\Delta_{o p t}$

a. Compare the value of $d_{\min }^{i}(\mathbf{H})$, and select the local optimal spatial modulation constellation $\Delta_{i}^{o p t}$ which has maximum $d_{\min }^{i}(\mathbf{H})$ as the optimal spatial modulation constellation $\Delta_{o p t}$.

(4)Feedback

a. Feed the information of $\Delta_{o p t}$ back to sender. In the next transmission, the sender uses the optimal spatial modulation constellation to map information bits.

Compared to AGSM, although the performance gain of L-AGSM algorithm decreases slightly, the computational complexity of L-AGSM algorithm is much lower than the proposed AGSM.

\section{Comparison of Computational Complexity for the Proposed Algorithm}

In this section, we evaluate the computational complexity of the proposed algorithm. For AGSM algorithm, there are $C_{N_{S}}^{N_{C}}$ candidate spatial modulation constellations, and each spatial modulation constellation needs to calculate Error! Reference source not found. times of Euclidean distance. Therefore, the calculation times of Euclidean distance imposed by AGSM algorithm is

$$
\delta_{A S G M}=C_{N_{S}}^{N} \cdot 2^{\left(\eta-\log _{2} N_{C}\right)} \cdot C_{N_{C}}^{2}
$$

However, in order to obtain $\Delta_{i}^{\text {opt }}$, it only needs to calculate $2^{\left(\eta-\log _{2} N_{C}\right)} \times$ $\left(\left(N_{C}-1\right) N_{S}-\sum_{j=1}^{N_{C}-1} j\right)$ times of Euclidean distance in L-AGSM algorithm, $i=1,2, \ldots, N_{S}$. So the calculation times of Euclidean distance imposed by L-AGSM algorithm is

$$
\delta_{L-A G S M}=N_{S} \cdot\left(\left(N_{C}-1\right) N_{S}-\sum_{j=1}^{N_{C}-1} j\right) \cdot 2^{\left(\eta-\log _{2} N_{C}\right)}
$$

Table 2 gives the calculation times of Euclidean distance when the spectral efficiency is $\eta=4 b i t / s / H z$ and $\eta=5 b i t / s / H z$. From Table 2, it is evident that the L - AGSM algorithm significantly reduces the computational complexity of AGSM in both cases. For example, when $\eta=4 b i t / s / H z$, the L-AGSM algorithm achieves a 71.77\% reduction in computational complexity compared with AGSM algorithm. 


\section{Table 2. Comparison of Computational Complexity between the Proposed AGSM and L-AGSM Algorithm}

\begin{tabular}{ccccc}
\hline $\begin{array}{c}\text { System } \\
\text { configuration }\end{array}$ & $\begin{array}{c}\text { Spectral } \\
\text { efficiency }\end{array}$ & $\begin{array}{c}\text { Spatial modulation } \\
\text { constellation point }\end{array}$ & $\begin{array}{c}\text { Complexity(the calculation } \\
\text { times of Euclidean distance) }\end{array}$ \\
$N_{T}=4, N_{A}=2$ & $\eta=4 \mathrm{bit} / \mathrm{s} / \mathrm{Hz}$ & 18 & 12240 & L-AGSM \\
& & & & 3456 \\
$N_{T}=6, N_{A}=2$ & $\eta=5 \mathrm{bit} / \mathrm{s} / \mathrm{Hz}$ & 45 & $8.64 \times 10^{8}$ & 23220 \\
\end{tabular}

\section{Simulation Results and Discussion}

In order to verify the validity of proposed algorithm and compare the performance of the proposed scheme with AGSM and L-AGSM, Monte Carlo simulation results are presented. In the simulations, the channel condition is assumed to be perfectly known at the receiver, and the feedback delay is zero. Raleigh fading channel with AWGN is assumed in all simulations, and $N_{R}=4, N_{A}=2$ is configured for GSM systems.

Figure 3 gives the BER performance curves of the proposed algorithms and existing algorithms when $\eta=4 \mathrm{bit} / \mathrm{s} / \mathrm{Hz}$. In the SM system, the number of transmit antennas is four and modulation scheme is QPSK. From Figure 3, it can be seen that AGSM algorithm performs better than GSM by about $4 \mathrm{~dB}$ performance gain at $B E R=10^{-5}$, while L-AGSM algorithm performs better than GSM by about $3 \mathrm{~dB}$ performance gain at $B E R=10^{-5}$. In addition, AGSM and L-AGSM also obtain $2 \mathrm{~dB}$ and $3 \mathrm{~dB}$ performance gain over SM, respectively. The important conclusion what Figure 3 reveals is that, under the same spectral efficiency, AGSM algorithm and L-AGSM algorithm are able to provide a considerable performance gain over GSM system.

The BER performance comparison between the proposed AGSM and L-AGSM is illustrated in Figure 4, and two spectral efficiency, $\eta=4 \mathrm{bit} / \mathrm{s} / \mathrm{Hz}$ and $5 \mathrm{bit} / \mathrm{s} / \mathrm{Hz}$ are considered. For both configurations, the BER performance of the proposed algorithms outperforms conventional GSM. But the L-AGSM algorithm is a local optimization algorithm, and doesn't fully use all possible generalized spatial modulation constellations. Therefore, the BER performance of the L-AGSM algorithm must worse than AGSM algorithm. However, the Figure 4 shows that the BER performance of L-AGSM the algorithm only decreases $1 \mathrm{~dB}$ compared to the AGSM algorithm in two cases. As we can see from the simulation results and Table 2, the L-AGSM algorithm can provide a better trade-off between computational complexity and performance. 


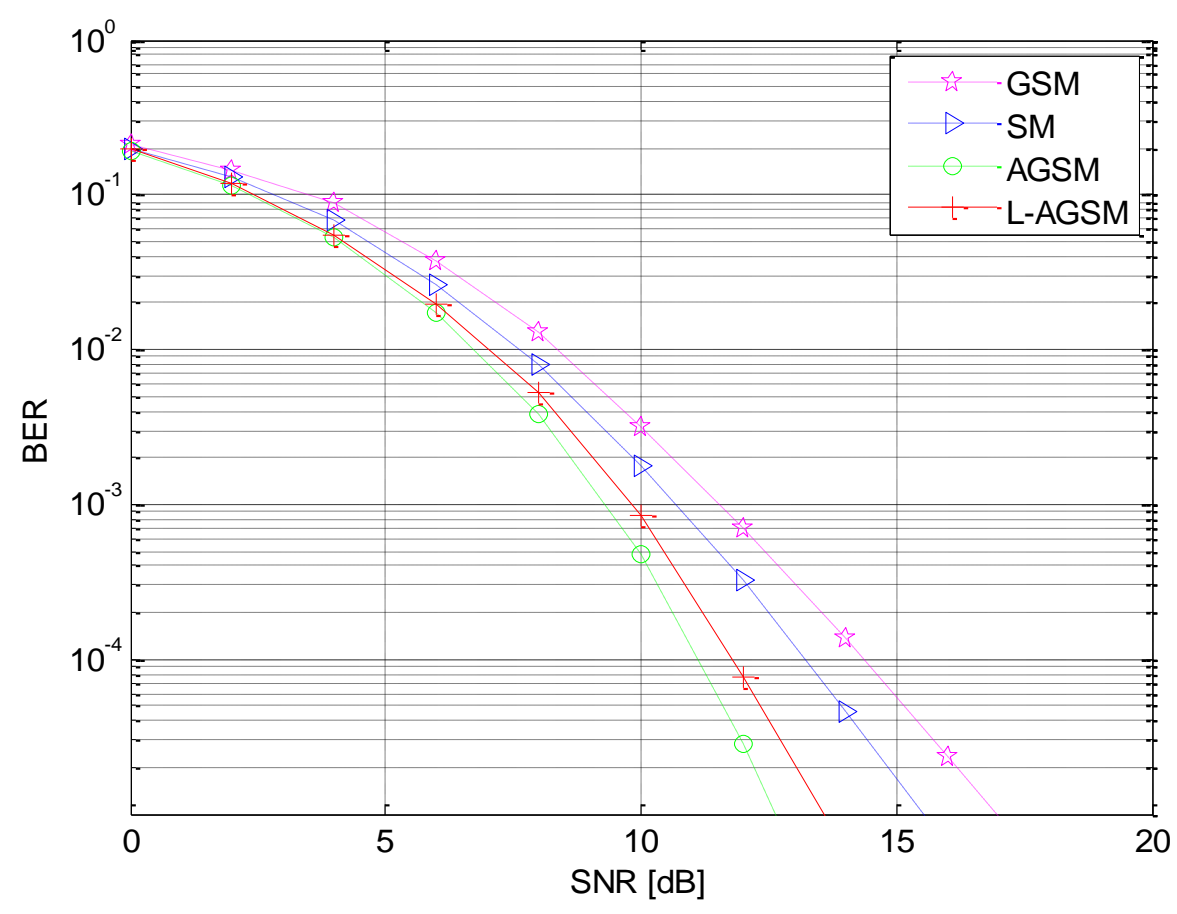

Figure 3. BER Performance Comparison between Proposed Algorithm and Existing Algorithm

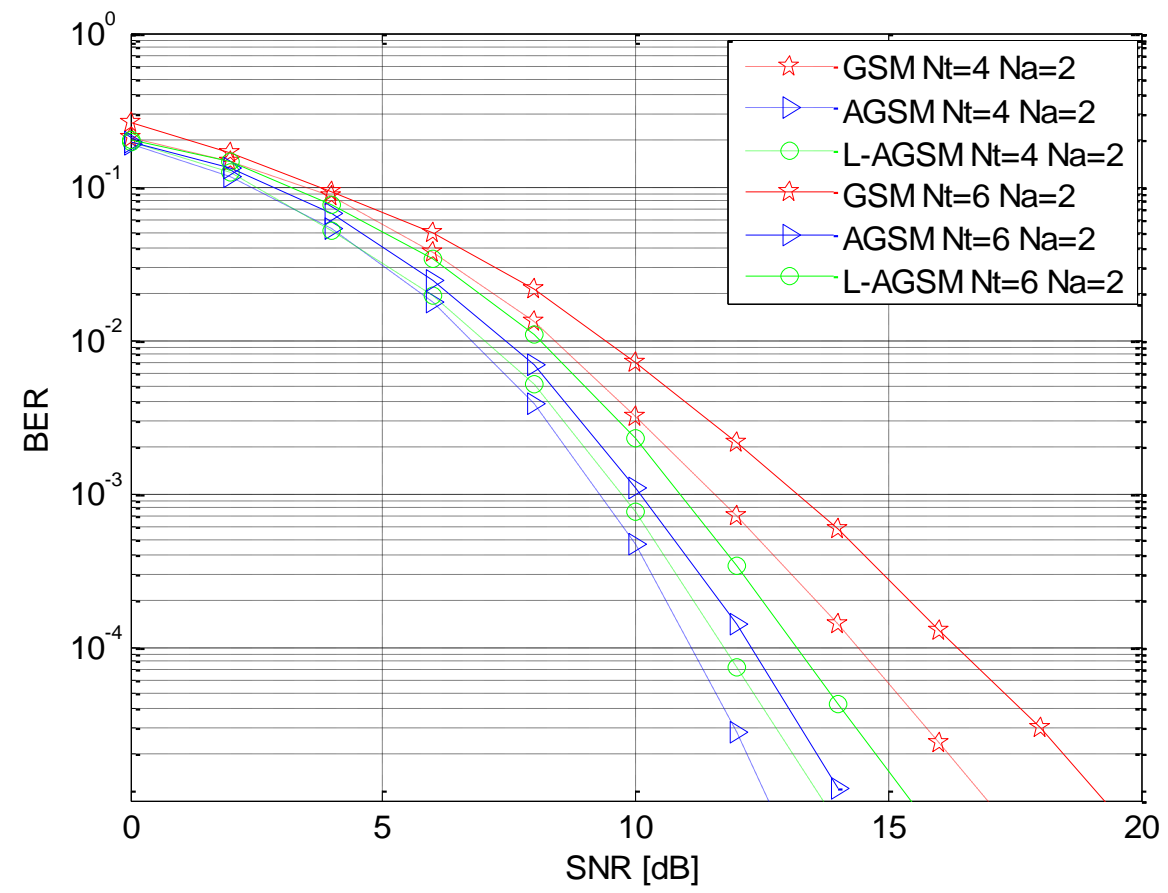

Figure 4. BER Performance Comparison between AGSM And L-AGSM 


\section{Conclusion}

In this paper, an AGSM algorithm and a L-AGSM algorithm are proposed to improve the BER performance of GSM. Monte-Carlo simulations demonstrated that the proposed algorithms are able to outperform GSM in BER performance. Furthermore, the computational complexity of the proposed algorithms is analyzed in this paper. From the analysis results, it is evident that L-AGSM can substantially reduce the computational complexity of AGSM while achieving the near BER performance compared to AGSM. Hence, the L-AGSM can be a promising transmission scheme for future MIMO systems.

\section{Acknowledgment}

We would like to thank Professor Dan Wang for her valuable comments and suggestions for improving the presentation of this paper. We also would like to thank the editor and reviewers for their hard work. This work was supported in part by the Basic and Frontier Projects in Chongqing under Grant No.cstc.2016jcyjA0209.

\section{References}

[1] M. D. Renzo, H. Haas, A. Ghrayeb, S. Sugiura, and L. Hanzo, "Spatial Modulation for Generalized MIMO: Challenges, Opportunities, and Implementation," Proceeding of the IEEE, vol. 102, no. 1, (2014), pp. 6-70.

[2] R. Mesleh, H. Haas, C. W. Ahn and S. Yun, "Spatial modulation-a new low complexity spectral efficiency enhancing technique", 1st International Conference on Communications and Networking, China, (2006).

[3] P. Yang, M. D. Renzo, Y. Xiao, S. Q. Li, and L. Hanzo, "Design guidelines for spatial modulation," IEEE Communications Surveys \& Tutorials, vol. 17, no. 1,(2015), pp. 6-26.

[4] J. Fu, C. Hou, W. Xiang and L. Yan, "Generalized spatial modulation with multiple active transmit antennas", Globecom Workshops, Miami, FL, USA, (2010), pp. 839-844.

[5] M. D. Renzo, H. Haas and A. Ghrayeb, "Spatial modulation for multiple antenna wireless systems: a survey”, IEEE Communications Magazine, vol. 49, no. 12, (2011), pp. 182-191.

[6] K. Ntontin, M. D. Renzo, A. Perez-Neira, and C. Verikoukis, "A low complexity method for antenna selection in spatial modulation systems", IEEE Communications Letters, vol. 17, no. 12, (2013), pp. 2312-231.

[7] R. Rajashekar, K. V. S. Hari, and L. Hanzo, “Antenna selection in spatial modulation systems," IEEE Communications Letters, vol. 17, no. 3, (2013), pp. 521-524.

[8] N. Pillay and H. Xu, "Comments on "Antenna selection in spatial modulation systems", IEEE Communications. Letters, vol. 17, no. 9, (2013), pp. 1681-1683.

[9] K. Ntontin, M. D. Renzo, A. I. Pérez-Neira, and C. Verikoukis, "A low complexity method for antenna selection in spatial modulation systems", IEEE Communications letters, vol. 17, no. 12, (2013), pp. 2312-2315.

[10] P. Yang, Y. Xiao, Y. Yu, and S. Q. Li, “Adaptive spatial modulation for wireless MIMO transmission systems", IEEE Communications Letters, vol. 15, no. 6, (2011), pp. 602-605.

[11] P. Yang, Y. Xiao, Y. Yu, and S. Q. Li, "Simplified adaptive spatial modulation for limited-feedback MIMO systems", IEEE Transactions on Vehicular Technology, vol. 62, no. 6, (2013), pp. 2656-2666.

[12] P. Yang, Y. Xiao, Y. Yu, and S. Q. Li, "Power allocation aided spatial modulation for limited feedback MIMO systems", IEEE Transactions on Vehicular Technology, vol. 64, no. 5, (2015), pp. 2198-2204.

[13] P. Yang, Y. Xiao, S. Q. Li and Y. Yu, "A Low-Complexity Power Allocation Algorithm for MultipleInput-Multiple-Output Spatial Modulation Systems", IEEE Transactions on Vehicular Technology, vol. 65, no. 3, (2016), pp. 1819-1825. 


\section{Authors}
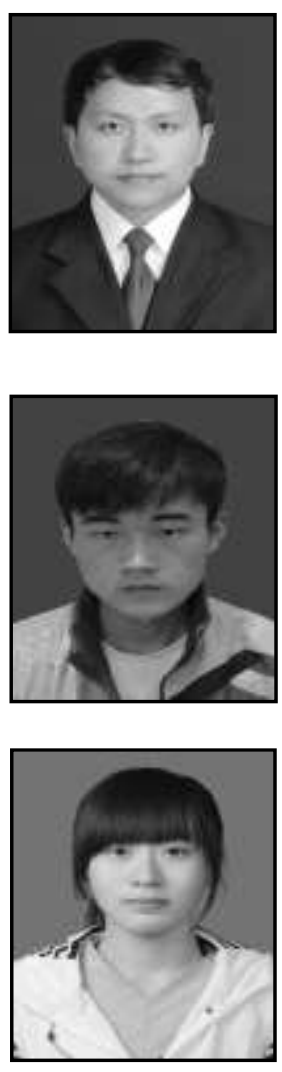

Fa-tang Chen, was born in Chongqing City, China, in 1965. He received the B.S. degree in maths from Jilin University, China, in 1988, and received the M.S. degree in applied mathematics from Beijing University of Posts and Telecommunications, Beijing, China, in 1999. Currently, he works as full professor at Chongqing University of Posts and Telecommunications. His research interests include physical layer algorithm in mobile communication system, and $5 \mathrm{G}$ wireless communication.

Fan-chao Zha, was born in Anhui Province, China, in 1991. He received the B.E. degree in electronic and information engineering from Bengbu College, Bengbu, China, in 2014. He is currently pursuing the M.E. Degree. His research interests include space-time coding, physical layer algorithm, and 5G wireless communication.

Han-yan Zhang, was born in Sichuan Province, China, in 1993. He received the B.E. degree in communication engineering from the Chongqing University of Posts and Telecommunications, China, in 2014. She is currently pursuing the M.E. degree. His research interests include HARQ technology and signal processing in wireless communications system. 
International Journal of Signal Processing, Image Processing and Pattern Recognition Vol. 10, No. 7 (2017) 